\title{
Peripheral Nerve Blocks: A Color Atlas
}

\author{
Jacques E. Chelly. Lippincott Williams \& Wilkins, Philadelphia, 2008, 3rd edition, \\ 496 pp, ISBN 978-0-7817-6876-4
}

Ivan Hsia, MD

Published online: 19 February 2009

(C) Canadian Anesthesiologists' Society 2009

As a senior resident transitioning into independent practice, I review this book amid advances in postgraduate educational curricula which aim to incorporate the latest innovative methods of regional anesthesia.

This third edition is comprised of 72 chapters that are divided into seven distinct sections. Section 1 details the general concepts regarding peripheral nerve blocks, including a general approach to the patient and a description of the essential tools (local anesthetics, nerve stimulators, and properly insulated nerve block needles). New to this section is an account of nerve mapping in adults, including several transcutaneous techniques. Section 2 describes single-injection peripheral blocks in adult patients and is subdivided into sections pertaining to the upper limb, the lower limb, and miscellaneous blocks. The miscellaneous block section includes, among other topics, cervical blocks, nerve blocks for the airway, and blocks of the head and face. Section 3 provides instructions on performing continuous nerve blocks in adult patients. Welltimed, relevant, and new to this edition is Section 4 which is dedicated to ultrasound. This section highlights performing nerve blocks by means of ultrasound. The text then shifts to pediatric patients, and Sections 5 and 6 review single-injection and continuous nerve blocks in children. Section 7, the last section of the text, explores the use of peripheral nerve blocks in managing chronic pain. Examples of some of the blocks presented in this section are the stellate ganglion block, the celiac plexus block, and facet joint blocks. A detailed listing of the topics covered in each chapter can quickly be retrieved with some clicking

I. Hsia, MD ( $\square)$

University of Ottawa, Ottawa, ON, Canada

e-mail: ivan.hsia@utoronto.ca through the navigation tabs of the book's related webpage, www.peripheralnerveblocks.com.

This textbook is not a definitive guide to all topics regarding regional anesthesia. Its primary purpose is to serve as an atlas relating to peripheral nerve blockade. Fittingly, not one neuroaxial technique is mentioned; instead, the atlas is filled with particulars regarding peripheral nerve blocks, and the text is designed in a stepby-step framework that facilitates performing nerve blocks. The text also contains numerous excellent photographs of real surface anatomy as well as carefully planned diagrams which are necessary for learning the spatial-motor tasks needed for performing nerve blocks. The text includes the following relevant information for each block type: block indications, optimal patient positioning, operator positioning, block needle, recommended local anesthetic, drug concentration and volume, relevant anatomical landmarks, and operator approach and technique. There is a 'helpful tips' section at the end of the description of each nerve block procedure that makes it easy to appreciate how to perform and 'trouble shoot' each technique. Whenever appropriate, nerve stimulation techniques are described for the blocks. Unfortunately, there is no description of stimulating catheters in the continuous catheter technique section. The images in the ultrasound section are clear and relevant to the procedures; this section also provides sideby-side images of the correct ultrasound probe placement, a cross-section of the relevant functional anatomy, and a picture of the ultrasound image. This methodology is ideal for learning about the "ultrasound anatomy" for the nerve blocks.

There are many peripheral nerve blocks and many approaches to each block. This text would be appropriate for residents learning these techniques and could be a useful reference for more experienced clinicians. The 
related online textbook provides access to the full version of the text and all related images, delivering ready access to the locations where blocks are performed. For its stated purposes, Peripheral Nerve Blocks: A Color Atlas 3rd Edition is an exceptional reference book with up-to-date information in the field of peripheral nerve blocks. 